\title{
ASPECTS SOCIO- ET PSYCHOLINGUISTIQUES DE LA MODÉLISATION DE LA COMPRÉHENSION DES TEXTES DE LA VIE QUOTIDIENNE: FAIT DIVERS ET PUBLICITÉ
}

\begin{abstract}
A bstract. Miczka Ewa, Aspects socio- et psycholinguistiques de la modélisation de la compréhension des textes de la vie quotidienne: fait divers et publicité [Social and psychological aspects of the modelling of understanding texts in everyday life. Various local news and publicity]. Studia Romanica Posnaniensia, Adam Mickiewicz University Press, Poznań, vol. XXV/XXVI: 2000, pp. 223-234, ISBN 83-232-0965-0, ISSN 0137-2475.

The author's aim is to study understanding of text as process, within text linguistics, with consideration of the main factors which condition it. The author concentrates her attention mainly on the phenomenon of the reader's constant referring to the text which he interprets to the information previously acquired by him.
\end{abstract}

\section{INTRODUCTION}

Le présent travail se situe dans le courant de la linguistique textuelle qui, premièrement, se concentre sur les mécanismes linguistiques permettant d'établir la cohérence locale et globale de la signification dans le texte et, deuxièmement, ou plutôt parallèlement, sur leur aspect ou dimension mentale. Nous pensons là au recours incessant aux connaissances préalables ou préconstruites du lecteur-interprétant. Troisièmement, nous tenons à introduire la perspective sociolinguistique en adoptant l'hypothèse que l'organisation des connaissances générales ou spécialisées est principalement le fruit des interactions sociales.

Notre exposé se divise en trois parties. Dans la première, nous allons situer le modèle de compréhension de texte proposé dans la perspective psycholinguistique. Ce modèle se base sur l'hypothèse que comprendre un texte signifie construire sa représentation. Dans la deuxième partie, nous présentons une de réponses possibles à ce problème: la représentation textuelle à quatre domaines: thématique, ontologique, fonctionnel et axiologique. La construction de la représentation textuelle est possible 
grâce aux schémas cognitifs. Parmi plusieurs formules proposées par les psycholinguistes, spécialistes en Intelligence Artificielle et sociologues ou sociolinguistes, nous avons choisi la notion de cadre de l'expérience introduite par Goffman en 1974 dans son ouvrage Frame Analysis (Goffman, 1974, 1991). Et la troisième partie de cet article sera consacrée aux relations entre les données provenant de quatre domaines de la représentation textuelle et le modèle de situation, ce dernier étant formulé en termes de cadres de l'expérience. Nous voudrions montrer l'utilité de cette notion dans la modélisation de la compréhension de texte à la base d'exemples; il s'agit des textes tels que publicité et fait divers.

\section{MODÈLE DE COMPRÉHENSION DE TEXTE DANS LA PERSPECTIVE PSYCHOLINGUISTIQUE}

Dans les opérations cognitives du traitement de texte, les psycholinguistes distinguent trois phases:

- la phase d'entrée qui inclut la compréhension et la mémorisation,

- la phase de conservation en mémoire,

- la phase de sortie qui embrasse la récupération de l'information sémantique et la production d'un nouveau texte.

Le modèle proposé concerne uniquement la première étape de la phase d'entrée: la compréhension. Quelles sont les opérations cognitives partielles nécessaires à la compréhension de texte? Denhière et Baudet indiquent les opérations suivantes: analyse syntaxique, récupération en mémoire des signifiés, construction des propositions psychologiques et établissement de leur cohérence locale, établissement de la cohérence globale de la signification et récupération en mémoire des connaissances préalables (Denhière, Baudet, 1992: 145). Les deux auteurs soulignent que les modèles de compréhension se basent sur deux hypothèses distinctes sur la nature de la compréhension. En s'appuyant sur ce critère, ils distinguent les modèles d'activation et les modèles à l'instanciation des schémas. Dans le cas des modèles d'activation, la compréhension «consiste à activer les connaissances - envisagées comme des portions d'un réseau d'associations - et à établir de nouvelles associations entre les noeuds du réseau» (op. cit.: 148). Les auteurs y classent le modèle symbolique d'Anderson et le modèle connexioniste de Rumelhart. Dans le second cas, celui des modèles à l'instanciation des schémas, la compréhension exige «l'intervention de connaissances décrites comme des structures de donnés préconstruites et disponibles en mémoire qui, une fois activées. contraignent la construction de la représentation» (op. cit.: 147).

Le modèle proposé se concentre sur la première phase du traitement de texte; la compréhension. Il se situe dans la classe de modèles à l'instanciation des schémas parce que nous postulons l'organisation des connaissances activées pendant la lecture en cadres de l'expérience. 


\section{COMPRÉHENSION DE TEXTE EN TANT QUE CONSTRUCTION DE LA REPRÉSENTATION TEXTUELLE}

Le texte est défini comme une suite phrastique qui permet au moins une des opérations suivantes: repérage du thème global, établissement d'idée directrice, élaboration du plan, résumé ou synthèse de deux ou plusieurs suites phrastiques.

Les processus cognitifs activés pendant la lecture aboutissent à la construction d'une représentation mentale du texte qui est, au moins partiellement, la reconstruction du programme minimum de l'émetteur. Nous envisageons le programme textuel minimum comme la constellation des choix qui visent:

- la situation modelisée dans le texte, ce qui impose un certain thème global (TG) et délimite le domaine thématique dans lequel le texte sera situé,

- la fonction dominante du texte,

- le registre textuel - le trait du texte distingué à la base du critère énonciatif qui est la structure des relations entre le locuteur et le(s) lectuer(s) inscrite dans le texte,

- le type de monde textuel: imitant la réalité «standard» ou créant un nouvel univers,

- l'orientation axiologique du texte résultant de la décision qui porte sur la prédication valorisante attachée au thème global.

Voilà le tableau qui présente le programme textuel minimum: ses constituants et les faisceaux des possibilités dans lesquels les choix sont effectués:

Tableau 1

\begin{tabular}{|l|l|}
\hline Constituents du programme textuel minimum & \multicolumn{1}{c|}{ Faisceaux de possiblilités } \\
\hline $\begin{array}{l}\text { 1. Situation modélisée dans le texte - thème } \\
\text { global (TG) }\end{array}$ & $\begin{array}{l}\text { a) objet ou ensemble/collection d'objets abstraits ou } \\
\text { concrets p. ex: chose, personne, animal, lieu, temps, } \\
\text { b) état, événement, processus }\end{array}$ \\
\hline 2. Fonction dominante & décrire, narrer, argumenter, instruire, expliquer \\
\hline 3. Registre textuel & $\begin{array}{l}\text { a) littéraire } \\
\text { b) non littéraire: institutionnel ou non institutionnel }\end{array}$ \\
\hline 4. Type de monde textuel & $\begin{array}{l}\text { a) non fictif } \\
\text { b) fictif: standard, non standard ou hybride }\end{array}$ \\
\hline 5. Orientation axiologique & $\begin{array}{l}\text { a) valorisation positive ou négative de TG } \\
\text { b) absence de valorisation }\end{array}$ \\
\hline
\end{tabular}

Nous admettons que pour comprendre un texte le lecteur doit prendre les décisions dans quatre domaines: thématique, ontologique, fonctionnel et axiologique. Il crée ainsi la représentation textuelle (RT) qui se compose de deux parties. La première partie contient les réponses aux questions engendrées par quatre domaines obligatoires de la RT, la seconde embrasse les procédures qui permettent d'obtenir ces réponses. Dans cet article nous nous concentrons sur la première partie de la représentation textuelle. 


\subsection{DOMAINE THÉMATIQUE DE LA REPRÉSENTATION TEXTUELLE}

Le premier domaine - thématique engendre les questions concernant les objets choisis comme thèmes: thèmes globaux, thèmes de composantes supraphrastiques et thèmes de phrases (Miczka, 1992) et les traits et/ou les relations attribués aux objets-thèmes dans le texte. Les réponses sont organisées dans le modèle de la structure thématico-rhématique modifiée par rapport aux modèles linéraires proposés par les linguistes tchèques (Danes 1974, Cervenka 1974). Nous postulons la structure thème-rhème suivante:

Tableau 2

\begin{tabular}{|l|l|}
\hline \multicolumn{1}{|c|}{ Organisation thématique } & \multicolumn{1}{c|}{ Organisation rhématique } \\
\hline $\begin{array}{l}\text { 1. thème global (TG) - dont on dérive les TCS } \\
\text { 2. thèmes de composantes supraphrastiques } \\
\text { (TCS) décrits par des ER }\end{array}$ & $\begin{array}{l}\text { ensembles rhématiques (ER) - faisceaux de rhèmes } \\
\text { attachés directement (rhèmes propres) ou } \\
\text { indirectement (rhèmes communs et empruntés) } \\
\text { à un TCS }\end{array}$ \\
3. thèmes de phrases & rhèmes de phrases \\
\hline
\end{tabular}

Pendant la première approche du texte il n'est pas nécessaire de saisir la structure thématico-rhématique de chaque phrase séparément; il est plus important de savoir repérer le thème global et les thèmes de composantes supraphrastiques, d'être capable d'assigner les ensembles rhématiques (ER) à ces thèmes et de (re)constituer l'ordre interne de chaque ER: spatial, temporel, ensembliste, axiologique ou événementiel.

\subsection{DOMAINE ONTOLOGIQUE DE LA REPRÉSENTATION TEXTUELLE}

Le deuxième domaine - ontologique définit les rapports entre l'univers textuel et la réalité. Le lecteur se pose la question si les faits relatés dans le texte sont situés dans la réalité standard. Au cas où la réponse serait positive, le lecteur peut intepréter le texte comme relatant fidèlement un fragment de cette réalité. «Fidèlement» signifie ici en accord avec les connaissances de l'auteur au moment de la production textuelle et suppose sa bonne foi. On peut y citer les entrées dans les encyclopédies, les notices d'emploi ou, théoriquement au moins, les livres scolaires. Même si le texte est catégorisé comme respectant les règles de la réalité standard, le lecteur peut décider qu'il s'agit là d'une transformation consciente d'un fragment de cette réalité où certains trains sont effacés, d'autres mis en relief p. ex: dans la publicité, l'horoscope, le curriculum vitae, la critique ou l'exposé politique. Un autre cas important est à noter; le texte qui dépasse les frontières de la réalité perçue comme standard. Le lecteur doit prendre la décision portant sur le statut ontologique des objets et sur celui des jonctions: objet - traits/relations qui sont assignés à cet objet dans le texte. Est-ce un 
univers textuel imaginaire ou onirique (récit de rêves, poésie), fantastique (science fiction, fantasy), merveilleux (contes de fées) ou mythologique/légendaire? Et, une question très importante, est-ce un univers homogène ou hybride - unissant deux ou plusieurs types de mondes fictifs?

En premier lieu, le repérage du statut ontologique de l'univers textuel se fonde sur la catégorisation des thèmes. Le lecteur classe les thèmes (global, de composantes supraphrastiques et phrastiques) soit comme les objets, événements, états, processus appartenant au monde qu'il connaît de son expérience quotidienne, soit comme quelque chose qu'il n'arrive pas à y localiser. Dans ce dernier cas, avant même d'évaluer les rhèmes, il peut formuler l'hypothèse concernant le type de monde non standard parce qu'il y a des thèmes typiques pour certains univers fictifs non standard $p$. ex: pour les contes, les nouvelles fantasy ou science fiction.

En second lieu, les décisions ontologiques du lecteur se basent sur l'évaluation des prédications attachées aux thèmes. Et si la comparaison des faits relatés dans le texte aux configurations factuelles du monde standard s'avère insatifaisante (manque de correspondance), le lecteur a recours à une autre comparaison. Il cherche des analogies dans les événements, états, processus caractéristiques pour certains univers non standard et transmis le plus souvent par certains types de textes, comme p. ex: la recherche des artifacts magiques est typique pour les textes fantasy, l'intervention des fées pour les contes, les compétences linguistiques des animaux pour les fables etc.

Les deux procédures sont nécessaires parce que, même si un thème est catégorisé comme appartenant à la réalité standard, la partie rhématique peut changer cette catégorisation et le situer dans le monde non standard. Le domaine ontologique concerne donc la catégorisation du monde créé dans le texte comme non fictif ou fictif. Dans ce dernier cas le lecteur doit décider s'il s'agit d'un monde fictif standard, non standard (fantastique, merveilleux, surréaliste) ou d'un monde hybride où les éléments de deux types de mondes se réunissent. En construisant ce domaine de la représentation textuelle le lecteur s'appuie sur les indices linguistiques: les prédicats-constructeurs du monde (rêver, s'imaginer, admettre, supposer etc.), certaines formules figées introductrices à un monde nons standard (p. ex: il était une fois), le changement du champ d'application des prédicats (p. ex: les animaux qui parlent, les arbres qui marchent, les pierres précieuses qui pensent etc.) et l'emploi des déterminants. Il faut souligner le rôle des descriptions définies qui présupposent l'unicité existencielle de l'objet auquel elles réfèrent. Ainsi donc «(...) en choississant une description définie particulière, le locuteur impose aux choses une grille à travers laquelle il les conçoit» constate De Mulder dans son article sur le rôle de l'article défini an tant que marqueur de l'évidentialité (De Mulder 1994: 109). La tâche cognitive du lecteur consiste à recueillir ces indices de l'ordre ontologique de l'univers textuel et à (re)construire une image qui n'est pas nécessairement homogène (la possibilité des mondes hybrides), mais qui satisfait à la condition de non contradiction. 


\subsection{DOMAINE FONCTIONNEL DE LA REPRÉSENTATION TEXTUELLE}

Le domaine fonctionnel engendre les questions qui portent:

- sur la fonction dominante du texte donc sur le type de réaction visé par l'auteur; le lecteur essaie de découvrir et d'interpréter les instructions qui concernent sa réaction future,

- sur le type de structures textuelles qui se manifestent dans le texte (argumentatives, descriptives, narratives, explicatives, instruction) (Werlich 1976, Van Dijk 1983, Adam 1996); il s'agit d'indiquer la structure supérieure dans le cadre de laquelle d'autres types de structures peuvent être exploités p. ex: les parties narratives ou explicatives dans le texte à visée argumentative (le récit d'une vie comme l'argument contre/pour un type de comportement), la description incrustée dans le texte narratif, ou la partie argumentative dans le texte principalement descriptif,

- sur le registre textuel; il s'agit d'inclure le texte dans une des catégories: texte littéraire, non littéraire, institutionnel, non institutionnel,

- sur l'énonciateur; le lecteur précise le statut de l'auteur en prenant en considération un faisceau de critères: institutionnel, individuel, rôle social et/ou professionnel, savoir présupposé, groupe d'âge, et établit une relation entre le personnage de l'auteur ainsi construit et lui-même: il peut s'identifier à l'énonciateur, se voir supérieur, inférieur, égal mais différent,

- sur le public construit dans le texte; une partie de la réponse est déjà fournie au moment d'établir une relation entre l'auteur et le lecteur - maintenant il s'agit de prendre en considération des indices tels que: lexique, domaines de connaissances que l'auteur suppose partager avec son lecteur prospectif, surtout l'ensemble des stéréotypes auquel l'auteur fait recours,

- sur l'emploi du texte- adéquat ou inadéquat à sa destination primaire p. ex: la poésie utilisée dans un texte publicitaire.

En répondant à ces questions le lecteur définit le type de texte conçu comme une constellation de traits de plusieurs niveaux: sémantique, syntaxique, pragmatique, rhétorique (Kerbrat-Orecchioni, 1980).

\subsection{DOMAINE AXIOLOGIQUE DE LA REPRÉSENTATION TEXTUELLE}

Ce domaine définit la localisation des objets textuels, surtout du thème global et des thèmes de composantes supraphrastiques dans le système des valeurs dont le texte est, implicitement ou explicitement, le porteur. La toute première démarche consiste à repérer le système des valeurs; à saisir la distribution des marques positives et négatives ou, éventuellement, à constater l'absence de valorisation. Cette absence de valorisation peut constituer une valeur en elle-même. Le lecteur répond à la question quels faits relatés dans le texte sont évalués positivement ou négativement. Il reconstruit les procédures de valorisation: statique où la valeur assignée à un objet-thème 
ne change pas ou dynamique qui se caractérise par la gradation de la même valeur ou, quelquefois par le changement de l'orientation axiologique.

Le domaine axiologique est étroitement lié au domaine ontologique de la représentation textuelle. Deux cas distincts sont à noter. Tout d'abord, il y a des textes qui, selon le lecteur, observent les règles de la réalité standard et dans lesquels la valorisation doit être principalement fondée sur les stéréotypes propres à cette réalité. Les textes qui créent le(s) monde(s) qui s'éloigne(ent) de l'ordinaire constituent un cas plus complexe. Ils peuvent reprendre les valorisations typiques pour le monde standard, comme le font $\mathrm{p}$. ex: les textes qui aident à la socialisation des enfants -contes. Mais, souvent, ils unissent deux ou plusieurs systèmes axiologiques parmi lesquels on retrouve celui du monde standard et des systèmes plus ou moins originaux construits au besoin de l'univers créé dans le texte; c'est le cas fréquent dans la science fiction.

\section{MODÈLE DE SITUATION ET REPRÉSENTATION TEXTUELLE}

\section{1. RôLE DU MODĖLE DE SITUATION DANS LA COMPRÉHENSION DE TEXTE}

Van Dijk et Kintsch soulignent le rôle du modèle de situation dans la compréhension textuelle: «(...) to understand the text we have to represent what it is about. If we are unable to imagine a situation in which certain individuals have the properties or relations indicated by the text, we fail to undertand the text itself. If we do not understand the relations between the local facts and global facts to which the text refers, we do not understand the text. (...) Using konwledge in discourse comprehension means being able relate the discourse to some existing knowledge structure, which then provides a situation model for it» (Van Dijk \& Kintsch, 1983: 337).

La compréhension du texte est donc une tầche globale qui, au début, exige l'existence d'un ensemble de modèles de situations dans la mémoire du lecteur. Le lecteur interprète les solutions partielles distribuées dans quatre domaines de la représentation textuelle: reconstruction de la structure thématico-rhématique, définition du statut ontologique de l'univers textuel et du type fonctionnel de texte, repérage de son système axiologique, en cherchant les analogies dans les modèles de situations réelles ou fictives.

\section{2. MODÈLE DE SITUATION FORMULÉ EN TERMES DE CADRE DE L'EXPÉRIENCE}

L'ouvrage de Goffman «Frame Analysis» $(1974,1991)$ fournit des instruments conceptuels qui, originellement conçus pour interpréter la perception des événements de la vie quotidienne, peuvent être adaptés à la compréhension des textes de la vie 
quotidienne, p. ex: les publicités ou les faits divers qui, nécessairement, de par leur fonction sont parasites de la vie quotidienne. «(...) identifier un événement parmi d'autres - dit Goffman - c'est faire appel, en règle générale, et quelle que soit l'activité du moment, à un ou plusieurs cadres ou schèmes interprétatifs que l'on dira primaires parce que, mis en pratique, ils ne sont pas rapportés à une interprétation préalable ou «originaire». Est primaire un cadre qui nous permet, dans une situation donnée, d'accorder du sens à tel ou tel de ses aspects, lequel autrement serait dépourvu de signification» (op. cit.: 30).

Les cadres de l'expérience se divisent en deux grandes classes: cadres naturels qui permettent d'identifier les événements non pilotés et cadres sociaux grâce auxquels nous comprenons d'autres événements «animés par une volonté ou un objectif et qui recquièrent la maîtrise d'une intelligence; ils impliquent des agencements vivants, et le premier d'entre eux, l'agent humain» (op. cit.: 31 ).

L'ensemble des cadres primaires, naturels et sociaux, constitue la cosmologie, le système de croyances d'un groupe donné, activé pour rendre signifiant le moindre événement de la vie quotidienne. Sont communes aussi les procédures qui permettent les transformations des cadres primaires; modalisations et fabrications. La modalisation est un processus pendant lequel «une activité donnée, déjà pourvue d'un sens par l'application d'un cadre primaire, se transforme en une autre activité qui prend la première pour le modèle mais que les participants considèrent comme sensiblement différente» (op. cit.: 52). Types de modalisation: les faire-semblant (jeux, fantasmes, scénarios), les rencontres sportives, les cérémonies, les réitérations techniques (apprentissage d'une tâche, démonstration théorique et pratique, utilisation d'enregistrement, expérience), les détournements. La fabrication concerne «des efforts délibérés, individuels ou collectifs, destinés à désorienter l'activité d'un individu ou d'un ensemble d'individus et qui vont jusqu'à fausser leurs convictions sur les cours des choses» (op. cit.: 93). Ses types sont les suivants: fabrications bénignes (tours, canulars expérimentaux, canulars formateurs, épreuves décisives de la loyauté et du caractère, machinations protectrices, fabrications purement stratégiques) et fabrications abusives (directes, indirectes, illusions).

Si l'on admet, en suivant l'idée de Goffman, que les événements de la vie quotidienne sont compréhensibles grâce à ou par l'intermédiare des cadres de l'expérience, on peut postuler que le même principe s'applique à la compréhension des textes plongés dans la vie quotidienne, qui rapportent donc plus ou moins fidèlement les situations quotidiennes ou qui en profitent pour argumenter, instruire, expliquer.

Dans cette perspective, comprendre un texte signifie:

a) accomplir les tâches cognitives partielles - répondre aux questions engendrées par quatre domaines de la représentation textuelle,

b) identifier le cadre de l'expérience le plus proche des faits relatés dans le texte,

c) organiser les solutions/réponses partielles en un tout cohérent en appliquant le cadrage supposé le plus adéquat. 


\subsection{RECONSTRUCTION DES CADRES DE L'ÉXPÉRIENCE DANS LA COMPRÉHENSION DES PUBLICITÉS ET DES FAITS DIVERS}

Ces deux genres textuels reflètent une attitude différente envers les cadres de l'expérience sur lesquels le lecteur - interprétant se base pour comprendre globalement le texte.

Le fait divers reprend le cadre que nous appelons le cadre-source sans le reformuler. Par le cadre-source nous comprenons le cadre de l'expérience qui normalement appliqué aux événements vécus ou observés dans la vie quotidienne, cette fois-ci sert à comprendre les événements relatés dans le texte.

La publicité transforme le cadre-source. La reformulation textuelle des cadres de l'expérience peut avoir une étendue différente. Premièrement, la reformulation la plus radicale. Chaque cadre-source ouvre un certain nombre de places pour les dramatis paersonae - agents et patients typiques d'une activité, les objets regulièrement impliqués dans une situation donnée, le lieu et le temps normaux d'une activité ou d'un état (on fait le ski en hiver, on s'attend au retour des forces vitales en printemps, etc.). Or, il est possible que ces places soient remplies par des entités non prévues dans le cadrage initial. Ce cas n'est pas rare dans les textes publicitaires. Il est illustré par la publicité qui suit:

\section{Au dessus d'un certain Q. I, on choisit la nouvelle Primera}

Nouveau 2. 01. Turbo Diesel

Test 1: Par rapport à un système concentionnel de réflecteurs, (1) les phares de la Nouvelle NISSAN PRIMERA améliorent notre vision de $80 \%$, pourtant (2) le singe préfère la petite voiture rouge parce qu'elle est rouge. . . Test 32:(3) la Nouvelle NISSAN PRIMERA est la seule voiture dotée d'un châssis à sécurité active avec suspensions QF et QT, pourtant (4) le singe préfère toujours la petite voiture rouge à pédales. ... Test 140: (5) Toutes les. Nouvelles NISSAN PRIMERA sont dotées en série d'une double correction d'assise pour le conducteur, de l'Airbag et de l'ABS, mais (6) le singe préfère quand même la petite voiture rouge avec le jolie claxon. . . . Test 316: Même si (7) la Nouvelle NISSAN PRIMERA est disponible aujourd' hui avec le nouveau 2. 01. Turbo Diesel, (8) Bobo le singe fait encore une fois le mauvais choix. . . . (9) Conclusion: Soyez malin un simple essai vous suffira pour choisir la Nouvelle NISSAN PRIMERA (disponible aussi en rouge). (10) Nouvelle NISSAN PRIMERA à partir de $103900 \mathrm{~F}$.

(11) Nissan

On voit les choses. autrement

Le texte est accompagné d'un support icônique où l'image d'une grande Nissan noire est contrastée avec celle d'une petite voiture rouge conduite par un singe.

L'univers textuel fait appel à la situation de test expérimental. La partie argumentative imite la description de ce procédé scientifique. Ainsi le texte reflète l'un des cadres sociaux modalisés - la démonstration - décrite par Goffman comme «(...) réitérations d'activités en dehors de leur cadre habituel qui permettent de donner une image fidèle de leur déroulement» (op. cit.: 75). Mais l'univers fictif du texte ne suit 
que partiellement la réalité standard parce que la démonstration qui est elle-même un cadre social modalisé subit ici une fabrication: l'objet du test - une voiture jouet et l'exécuteur - Bobo le singe remplacent de vrais objet et agent typiques pour ce cadre.

Il faut noter d'autres types de reformulations textuelles de cadre-source, moins radicaux, qui gardent la structure générale du cadrage servant de base pour la situation modélisée dans le texte. Les places ouvertes par le cadre-source sont remplies par des agents, patients, objets, lieux et temps initialement prévus. Le changement porte sur leurs traits et/ou sur les traits des relations qui s'instaurent entre les dramatis personae (mais les relations elles-mêmes sont reprises intactes du cadrage initial). Ces traits peuvent être stéréotypisés à l'extrême; c'est le cas où le publicitaire introduit les agents stéréotypisés qui, selon lui, reflètent les types d'utilisateurs d'un produit donné. Ensuite, le cadre-source peut subir soit l'effet d'hyperbole - les traits des composants du cadre ou des relations entre eux sont intensifiés, soit l'effet de litote où certains traits sont consciemment affaiblis. Le tableau ci-dessous présente la synthèse des reformulations textuelles d'un cadre-source:

Tableau 3

\begin{tabular}{|c|c|}
\hline \multicolumn{2}{|c|}{ Reformulations textuelles de cadres de l'expérience } \\
\hline 1. la structure du cadre-source transformée & 2. la structure du cadre-source intacte \\
\hline $\begin{array}{l}\text { Les places ouvertes par le cadre-source sont } \\
\text { remplies par des entités non prévues initialement; } \\
\text { la substitution concerne: } \\
\text { a) dramatis personae, } \\
\text { b) objets, } \\
\text { c) temps et lieu }\end{array}$ & $\begin{array}{l}\text { Les traits des composants du cadre-source et/ou } \\
\text { le relations entre ceux-ci: } \\
\text { a) sont stéréotypisés, } \\
\text { b) subissent l'effet d'hyperbole, } \\
\text { c) subissent l'effet de litote }\end{array}$ \\
\hline
\end{tabular}

Les fait divers, comme nous venons de le signaler, reflètent une attitude différente envers le cadre-source. Ils reprennent le cadrage-source intacte. Ils jouent plutôt sur les relations entre deux ou plusieurs cadres de l'expérience; les cadres explicitement évoqués ou les cadres implicites. Parmi les relations organisant les cadres dans un seul texte, il faut mentionner:

- la relation de succession temporelle,

- la relation cause-conséquence,

- la relation d'exclusion,

- la relation d'inclusion.

En voilà des exemples. Le premier fait divers «Michael Jakcson toujours tête en l'ai » illustre la relation d'exclusion entre deux cadres de l'expérience explicites. Le caldre suggéré dans la troisième phrase «tester un nouvel uniforme antipollution» est rejeté par l'introduction d'un cadre nouveau - «s'initier au parachute ascensionnel». Le texte est accompagé par la photo de Michael Jackson en combinaison.

\section{Michael Jackson toujours tête en l'air}

(1) Une petite devinette: quelle star internationale se cache derrière cet accoutrement digne d'un monstre du Loch Ness? (2) Mais oui, il s'agit bien de Michael Jackson! (3) Et contrairement 
à ce qu'on pourrait penser, la pop star n'est pas en train de tester un nouvel uniforme antipollution. (4) Elle a seulement voulu s’initier au parachute ascensionnel dans les îles Vierges. (5) Lunettes de choc. combinaison isolante. et hop! voilà Bambi se prenant pour un petit oiseau.

Ici Paris, 20-26. 05. 1998

Le texte suivant présente trois cadres de l'expérience explicites en relation d'inclusion. Le cadre global qui est un cadre social non modalisé - «accidents au bord des navires de croisière» embrasse deux cadres modalisés: le cadre de simulation «imitation de l'action exécutée par l'héroïne du film „Titanic” et le cadre de jeu d'acteur servant de base pour le cadre précédent».

(1) Titanic pourait bien detenir un nouveau record: celui des accidents! (2) Aux Etats-Unis, de plus en plus de passagers des navires de croisière tentent de se mettre debout. les hras en l'air. sur la proue du bateau. comme dans le film. (3) Mais, ce n'est pas Kate Winstlet qui veut!

Ici Paris, 20-26. 05. 1998

Le dernier exemple montre l'interaction entre le cadre explicite et le cadre implicite. Le cadre explicite - «édition d'une brochure par l'Ambassade de France à La Haye» serait incompréhensible si l'interprétant n'était pas capable de reconstruire un cadre implicite - «état d'opinions et d'attitudes des habitants des Pays-Bas envers les Français».

(1) L'ambassade de France à La Haye vient d'éditer à 500000 exemplaires une brochure à l'intention des touristes néerlendais: «Nous avons tous une douche, nous sommes aussi propres que vous, même les toilettes des autoroutes ont fait des progrès».

Le Monde, 30. 05. 1998

\section{CONCLUSION}

Ainsi donc, dans la perspective adoptée dans cet article, la compréhension du texte embrasse non seulement les réponses aux questions engendrées par quatre domaines de la représentation textuelle et leur organisation un en un modèle de situation compréhensible pour le lecteur. Celui-ci reconstruit en plus la relation entre la situation reconnue dans le texte et le cadre de l'expérience qui a servi de sa base. Pour que la compréhension soit réussie, l'interprétant devrait être capable de répondre à la question si le cadre-source a été repris intact dans le texte ou s'il a subi des transformations. Les transformations du cadrage original peuvent être structurelles: il s'agit là de la substitution des composants impliqués par le cadre-source par des éléments nouveaux, étrangers au cadre-source, ou qualitatives - quand les traits des composants du cadre-source ou des relations sont séréotypisés, intensifiés ou affaiblis. Et si, dans le texte, le lecteur découvre deux ou plusieurs cadres de l'expérience, il doit aussi prendre la décision concernant les relations qui les organisent en un modèle de situation. 


\section{BIBLIOGRAPHIE}

A d a m, J-M., (1992), Les textes: types et prototypes, Paris, Nathan.

B arthes R., (1964), Structure du fait divers, In: Essais critiques, Paris, Seuil, p. 188-197.

Cervenka M., (1974), O tematycznym nastepstwie, In: M. R. Mayenowa, Tekst i jezyk. Problemy semantyczne, Wrocław, Ossolineum, p. 85-97.

Charolles, M., (1989), Coherence as a Principle in the Regulation of Discourse Production, In: W. Heydrich et al. (eds.), Connexity abd Coherence. Analysis of Text and Discourse, Berlin, New York: W. de Gruyter, p. 5-15.

Coirier P., Gaonac'h D., Passerault J-M., (1996), Psycholinguistique textuelle. Approche cognitive de la compréhension et de la production des textes, Paris, Armand Colin.

Danes F., (1974), Semantyczna i tematyczna struktura zdania i tekstu, in: M. R. Mayenowa, Tekst i jezyk. Problemy semantyczne, Wrocław, Ossolineum, p. 23-40.

Denhière G., B audet S., (1992), Lecture, compréhension de texte et science cognitive, Paris, PUF.

De Mulder W., (1994), La «création du monde» par l'article défini. Le, marqueur évidentiel?, Langue Française 102, p. 108-120.

Dijk Van, T. A., Kintsch W., (1983), Strategies of Discourse Comprehension, New York, Academic Press.

Dubois D. (ed.), (1993), Sémantique et cognition. Catégories, prototypes, typicalité, Paris, CNRS Editions.

Ducrot, O., (1980), Les échelles argumentatives, Paris, Minuit.

Goffman, E., (1974), Frame Analysis. An Essay of the Organisation of Experience, traduction française: Les cadres de l'expérience, 1991, Paris, Minuit.

Grice, H. P., (1975), Logic and Conversation, in: P. Cole, J. L. Morgan (eds.), Syntacs and Semantics. Vol. 3: Speech Acts, New York: Academic Press, 41-58.

Grize, J-B., (1981), Pour aborder l'étude des structures du discours quotidien, Langue Française 50 , p. $8-23$.

Kerbrat-Orecchion i, C., (1980), Enonciation de la subjectivité dans le langage, Paris, Arman Colin.

Kleiber G., (1994), Contexte, interprétation et mémoire: approche standard vs cognititve, Langue Française 103, p. 9-22.

Klemensiewicz Z., (1982), O syntaktycznym stosunku nawiazania, in: A. Kalkowska (éd.), Sktadnia, stylistyka i pedagogika jezzykowa, Warszawa, PWN.

Miczka E., (1992), Les mécanismes sémantiques et rhétoriques de la cohérence du commentaire politique, Katowice, Editions de l'Université de Silésie.

Miczka, E., (1993), Les structures supraphrastiques dans le texte: procédures et analyses, Neophilologica 9, p. 41-60.

Miczk a E., (1996), Inférences dans la construction d'une représentation textuelle. Sur l'exemple d'un texte publicitaire, in: K. A. Sroka (ed.), Kognitive Aspekte der Sprache, Linguistische Arbeiten 360, Tübingen, Niemeyer, p. 181-187.

Petofi J., (1985), Procedural Aspects of Text Interpretation, in: E. Sozer (ed.): Text Connexity, Text Coherence. Aspects, Methds, Results, Hamburg: H. Buske, p. 176-188.

Werlich E., (1976), A Text grammar of English, Heidelberg. 